cost effectiveness, and (b) the value to morale of staff and patients of seeing this sort of turnover. Compulsory detention, as an end in itself, could be greatly reduced, which is the position that obtains for a number of patients after a few years in which treatment has been to no avail.

4. The prisons would benefit, having access to more beds more quickly. The beds currently blocked by treatment failure would be liberated more readily. Both prisons and Special Hospitals would benefit from an improved dialogue between them, with easier two-way movement of patients.

5. There would be an improvement of the ethos of Special Hospitals, away from the sometimes excessive emphasis on security and safety. These concerns are often initiated by the activities of a few embittered, untreatable patients with personality disorder who see no way out of hospital, and who have little to lose by disruptive behaviour, or worse.

6. The increased patient turnover would have obvious and beneficial effects to the research into aspects of treatment, nosology and aetiology of those with personality disorders. Without such research, especially into the various aspects of management, the future of the Special Hospitals in this respect must be considered in jeopardy.

The views expressed are solely those of the author and do not necessarily represent those of Broadmoor Hospital, the Institute of Psychiatry or the DHSS

\title{
Who Cares for the Adult Brain Damaged?
}

\author{
Jane Newson-Smith, Consultant Psychiatrist, Knowle Hospital, Hants
}

There is increasing interest in services for younger brain damaged persons (acquired in adult life as a result of trauma or illness). However, there is uncertainty about the type of service needed and to whom the medical responsibility belongs.

My own interest began in 1981 when I took over consultant responsibility for the Hamble Unit. This 28-bed unit opened at Knowle Hospital (Adult Mental Illness) in 1973 and serves the Southampton Psychiatric Health District. Patients had previously been placed in long-stay and psychogeriatric wards.

Over the last decade patients have been selected on the criteria of becoming brain damaged in adult life, requiring a high degree of both physical and psychiatric nursing and frequently having behavioural problems. Previously it was considered that they were not receiving optimal care and, furthermore, they were disruptive on other wards. The philosophy of Hamble Unit has always been to promote the highest possible quality of life in these grossly handicapped patients, and also to create and maintain as many links as possible with their families and the community. Initially the Unit was seen as a final placement, including terminal care. However, this meant that patients were denied admission in acute phases of behavioural disturbance after head injury, for example, and in recent years we have felt that the team's skills can be appropriate to selected patients who have considerable potential for recovery and rehabilitation. Furthermore, we have found it feasible to mix patient types and this is important for staff satisfaction.

Preliminary analysis of nearly a decade of experience in the unit shows that the following categories of patients have problems where nursing in a special brain damage unit is appropriate at some stage in their illnesses:

(1) Late stages of progressive dementing illnesses usually associated with physical frailness: e.g. Huntington's Chorea, Alzheimer's Disease. Disseminated Sclerosis with cerebral involvement; (2) Non-progressive (or not necessarily progressive) brain damage: e.g. alcoholic dementias, brain damage due to epilepsy, frontal lobe type syndromes after non-communicating hydrocephalus and cerebrocascular accidents; (3) Traumatic brain damage: (i) early, with behavioural problems and (ii) late-severely cognitively impaired patients, invariably with severe physical handicaps and communication disorder.

The unit has never attempted to be a sick ward to the hospital'. Patients in some of the above categories may at some stage be nursed on other wards when their awareness and cognitive functions are less impaired. Generally we find patients with progressive illness, particularly with involuntary movements, are more comfortable and less embarrassed on the unit.

It is difficult to gain an impression of the size of the problem, either on a national or local level. Certainly there are few specialist units. Occasional patients, after head injury with severe behavioural problems, are totally misplaced and can create exceptional havoc in a medical setting. Some Health Authorities are prepared to finance contract beds at specialist units (e.g. the Kemsley Unit for brain injury behaviour disorders at St Andrew's Hospital, Northampton) —others are not. National figures are not very helpful. The last in-patient census in mental hospitals was in 1971. An outline estimate from this shows that there were up to 4,840 patients with the characteristics of 'dementia before the senium' in mental illness beds in England and Wales, 
giving a ratio of between 12 and 20 beds needed per average Health District of 200,000. The mental health inquiry gives a figure of 801 admissions in 1980 in England, with a main (ICD9) diagnosis of presenile dementia. The main diagnosis is the initial diagnosis on admission to hospital, but many of these patients have multiple and altered diagnoses. In 1980 there were $\mathbf{7 4}$ admissions for alcoholic dementias, other than delirium tremens, and Korsakov's psychosis.

It does seem, nationally and regionally, that there is a lack of clear planning policies for these problem patients. Certainly, a proportion of severely cognitively impaired patients fall through the medical services and into a medical 'no man's land'. We currently need to look at the size of the problem in the community at district level. Often many different agencies are involved, with poor liaison. Follow-up psycho-social civilian literature on traumatically brain damaged patients is limited, but suggests that much of the burden is carried by families (Weddell et al, 1980). Relatives find cognitive impairment and behavioural problems much more difficult to cope with than physical handicap. Multidisciplinary Community Mental Handicap teams do take on a steady trickle of referrals, and their skills are often the most appropriate. Occasionally patients are admitted to Mental Handicap beds. Adult training centres may prove useful in the day time and provide some relief for relatives. Patients with both physical and behavioural problems (e.g. advanced disseminated sclerosis with frontal lobe involvement, leading to extreme sexual disinhibition) present occasional management difficulties to primary care community nurses. Nurses have reported difficulty in getting skilled management guidance. Patients are often known to local Social Service Departments and their community occupational therapists. Voluntary organizations may be involved, particularly COMBAT for Huntington's Chorea and HEADWAY for head-injured patients and their families. Frequently families are known to Child Guidance because of related disturbance in the home. Families of Huntington's Chorea victims have special problems and counselling needs. Registers are needed and genetical counselling (Martindale and Bottomley, 1980).

There is a lack of suitable medical in-patient facilities for the young chronic sick, and the brain damaged are likely to be excluded. It is a similar picture with hostel and resident home accommodation, both for long-term care and intermittent relief admissions. Some younger brain damaged patients are nursed inappropriately on medical geriatric wards. One particular problem concerns patients with severe brain damage who are totally dependent on physical nursing but do not require psychiatric nursing - an example is the chronic, vegetative state after trauma when the patient is awake but has no awareness. Some districts, such as our own, do have a policy that these patients remain the responsibility of acute medical/surgical teams. Many regional physical rehabilitation units are not able to cope with the very occasional extremely disruptive behaviour disturbance after brain trauma. Negative behaviour patterns may be reinforced by nursing attention to keep them quiet. Nevertheless, for the vast majority admission to a psychiatric hospital is inappropriate, denying them intensive physical rehabilitation. On a supra-regional level it would make sense to consider physical rehabilitation services with specialist psychiatric, psychological and psychiatric nurse input on a liaison basis. The post-trauma patients certainly need psychological understanding.

It is fundamental to consider whether an identified specialized psychiatric hospital service is required or whether an ad hoc arrangement between general psychiatry, psychogeriatrics and Mental Handicap suffices. Traditionally, the Mental Handicap services have cared for individuals who acquire their brain damage before the development of the mind is complete'. A dividing line at 18 years is a working rule. Professor D. J. Bicknell (personal communication) believes the dividing line was defined more clearly in the Acts that preceded the 1959 Mental Health Act. Some consultants in Mental Handicap are interested in this group of patients and are involved in providing a psychiatric service for them. Psychogeriatricians are sympathetic to the problems and the debate continues. Some consultants doubt that sufficient commitment exists in the Adult Services and feel that most cognitively impaired younger patients are best cared for in dementia services, while the behaviourally disturbed patient is best catered for in wards for disturbed, chronic psychotics. (Specialist cognitive rehabilitation and behavioural modification would, however, be difficult to deliver.) Others disagree, feeling that dementia services are geared for an age of 75 upwards and are not suitable for younger patients and their families, who get a relatively poor deal. Certainly, many of the younger patients tend to be over-active but with relatively intact parietal lobe function, requiring space and occupational therapy geared to younger adults.

I see no reason for a hard and fast rule of a psychogeriatrician, a general psychiatrist or a mental handicap psychiatrist having consultant responsibility, but would promote the idea of a specialist unit, with identified consultant(s). I have found the work provides an enjoyable contrast to adult psychiatry. If working substantially in dementia or amentia services. I feel I would want the contrast to be with non-cognitively impaired patients. However, if professional competency with these patients is to develop, it is essential for the psychiatrist in charge to be in continuing and frequent dialogue with colleagues in the other services. Many general psychiatrists still do not receive training in Mental Handicap which I personally have found to be a great disadvantage in adult brain damage work. We have found treatment of psychotic disturbance in grossly organically impaired patients to be particularly difficult as the presentation is so different from that in general psychiatric practice. Patients who do well (e.g. post-traumatic dementia patients and alcoholic dementias) may need to be referred 
back to general services for rehabilitation and, sometimes, long-stay provisions in hostels. The psychiatrist does need to have a regular commitment to the team and should take a leadership role.

Nurse selection is of prime importance. The work is physically and emotionally challenging, but certainly appeals to a sufficient number of excellent nurses. We have always had the principle that nurses may be allowed to move off the ward at short notice, without detriment to their career, if they find the work unsuitable. Adequate and sufficient staff levels are very difficult to maintain, and this has at times caused poor staff morale and conflict. Training in psychiatric nursing is the most common qualification, but registrations in general nursing and in mental subnormality are also very important. On a larger unit I feel that a nurse should be trained in behavioural modification. It is difficult on a shorter working week and shift system for the ward nurses to undertake the community nurse role with patients and relatives. However, they are the best nurses to do it, and in addition, this improves their job satisfaction for otherwise they are working predominantly with patients with limited language. Psychotherapeutic skills are essential as both brain damaged patients and their families have major losses which need grief work.

Psychologists are now leading the field in cognitive rehabilitation, particularly in North America. New techniques, such as use of microcomputers for re-learning, are being evaluated. Sessions of neuropsychology and behavioural modification are required. Normally nursing staff and occupational therapists can be responsible for the daily running of programmes. This is also a specialist field for occupational therapy, together with other medical therapists. An occupational therapist, with a specialist knowledge of physical handicap and cognitive impairment, is needed. Seemingly simple advice on eating aids, etc., can make a major difference to the dignity and independence of the patients. We have found speech therapy to be a scarce resource in the NHS and yet probably the most disabling handicap for our patients lies in communication. Speech therapy sessions are needed. Few doctors, including psychiatrists, ever receive much training in language problems. In fact, much of the early speech rehabilitation can be done under guidance by other members of the team. Behaviourally disturbed brain damage patients with aphasias may be too difficult for formal sessions and yet the balance of opinion shows that such patients do better with speech therapy. Familiarity with various forms of non-verbal language is important. Access to physiotherapy sessions is important but, again, nursing staff and occupational therapists can work many of the programmes under guidance. Family work is important and can be very challenging. Frequently marriages break down after severe cognitive impairment following head trauma, and the family dynamics in families with illnesses such as Huntington's Chorea need special expertise. Social Work input is needed both for patient and family work and also in a consultative capacity to other members of the staff.

My experience has been that special attention does need to be paid to staff morale. One is constantly faced with low staffing levels and also trying to ensure that only suitable staff, both in trained and non-training grades, are appointed to the ward. Nurses are needed who enjoy both the actual physical and psychiatric nursing of these patients, but who also wish to gain specialist skills in the psychotherapies, cognitive rehabilitation, etc. The ward can be made very noisy by only one or two problem patients, and tolerance levels decrease, particularly when staffing levels are minimal. Issues such as non-accidental injury to patients need to be talked about openly and constructively rather than just dismissed as something that happens in other hospitals. A continuing programme of staff education is important. Nurses and other therapists need to be recognized in their own hospital as specialists.

In summary, I do believe specialist units are important, possibly serving populations of around one million, i.e. subregional services. This compromises between optimal development of specialist skills and reasonable travelling distances for relatives, etc. Siting is best away from a busy, congested general hospital. Obviously spacious ground floor accommodation and gardens are needed, with emphasis on adequate soundproofing for some rooms. Facilities are needed for both long and short-term patients, intermittent admissions and day patients. The unit should be the base for community work, and liaison psychiatry should be a major role. Close relationships with Mental Handicap, dementia services and adult mental illness services have to be encouraged, with possibly all represented on the staff.

\section{REFERENCES}

Martindale, B. \& Bottomley, V. (1980) The management of families with Huntington's Chorea: A case study to illustrate some recommendations. Journal of Child Psychology and Psychiatry, 21, 343-51.

Weddell, R., Oddy, M. \& Jenkins, D. (1980) Social adjustment after rehabilitation: A two-year follow-up of patients with severe head injury. Psychological Medicine, 10, 257-63. 